\title{
IDENTIDAD REGIONAL Y CONDUCTA ELECTORAL: EL CASO DEL PAN EN YUCATÁN
}

\author{
Regional Identity and Electoral Behavior: A Case Study of the PAN Party in Yucatán \\ Jorge Enrique Figueroa-Magaña
}

Resumen: La "región" es una de las variables más importantes para explicar el comportamiento político mexicano. Sin embargo, no se sabe con precisión qué mecanismos se involucran en este concepto y cuáles son los "efectos regionales". Mediante los datos de una encuesta, este trabajo examina empíricamente si la identidad local yucateca guarda relación con el apoyo electoral histórico hacia el otrora opositor Partido Acción Nacional (PAN) en ese estado. Para tal fin, se proponen y analizan dos modelos: el modelo de atención cultural de lealtad panista y el modelo de efectos de política pública. El análisis estadístico revela poca evidencia para el primer modelo, pero sí un apoyo considerable hacia el segundo, en especial entre aquellos de mayor nivel educativo. Por tanto, la tradicional cultura yucateca ayuda a explicar por qué los yucatecos, aun los menos favorecidos económicamente, apoyan al conservador PAN.

Palabras clave: conducta electoral, identidad, voto panista, modelo de conciencia cultural, modelo de efectos de políticas públicas.

Abstract: The region is one of the most important variables to understand Mexican political behavior. However, little is known about how region shapes voting behavior and what mechanisms are involved in the "regional effects". Using data collected through a large scale survey, this article empirically examines one mechanism, the role of a Yucatecan regional culture in fostering opposition voting for the National Action Party (PAN) in the Mexican state of Yucatan. Two models are tested: the cultural awareness model of PAN loyalty, and a policy effects model of PAN loyalty. The data analysis revealed little evidence for the first model, but considerable support for the policy effects model, particularly among the highly educated. Thus, the traditional regional culture appears to be a factor in explaining why Yucatecans, even the more deprived, support the conservative PAN.

Keywords: political behavior, identity, panista vote, cultural awareness model, policy effects model.

Jorge Enrique Figueroa-Magaña, doctor en Ciencia Política por la Universidad de Kentucky. Profesor e investigador en la Universidad de Quintana Roo. Temas de especialización: regionalismo y nacionalismo mexicano, cultura y comportamiento político. Correo electrónico: jfigueroa@uqroo.mx.
Enviado a dictamen: 22 dejunio de 2012 Aprobación: 19 de septiembre de 2012 Revisiones: 1 


\section{Introducción}

L a literatura actual sobre conducta electoral mexicana a nivel macro identifica muchos factores que inciden en el ciudadano a la hora de acudir a las urnas y sufragar por un determinado partido político. Una línea teórica de corte económico/racional enfatiza el rol de evaluaciones prospectivas y retrospectivas de la economía nacional y personal hacia el partido en el poder, en concordancia con las expectativas de la teoría normal del votante económico (Camp, 2004; Buendía, 1996; Bruhn, 1999; Domínguez y McCann, 1996; Poiré, 1999; Magaloni, 1999; McCann, 1998). Una segunda línea teórica se enfoca en las dinámicas transitorias de una elección, como las campañas y la penetración, los atributos personales de los candidatos, el rol de los medios de comunicación y otros factores intrínsecos a una elección (Moreno, 1999, 2004; Domínguez, 2004; Klesner, 2004; Bruhn, 2004; Lawson, 2004a, 2004b).

En las últimas décadas, y con mayor frecuencia desde las elecciones presidenciales de 2000 y 2006, algunos politólogos se han enfocado en la variable "región" para explicar el voto ciudadano en México. Esta tendencia está basada en dos poderosas razones. Una, la alternancia política en México comenzó a nivel subnacional (Cornelius, Eisenstadt y Hindley, 1999); en segundo lugar, fueron las provincias mexicanas donde "el proceso electoral se aceptó como la única manera legítima de obtener y preservar el poder, aun antes de que ocurriese una democratización electoral a nivel nacional" (Díaz, 2004: 219). ${ }^{1}$ Sin embargo, poco se sabe de cómo esta variable moldea la conducta electoral del votante, qué mecanismos se involucran en el efecto "región", por qué emergen patrones partidistas en regiones geográficamente distantes y por qué la oposición al otrora hegemónico Partido Revolucionario Institucional (PRI) adquirió, de igual manera, tintes regionales en las últimas décadas.
El estado de Yucatán ejemplifica estas cuestiones. Sabemos que el Partido Acción Nacional (PAN) yucateco se convirtió, desde mediados de los años cuarenta del siglo pasado, en una gran fuerza opositora que incluso llegó a propinar algunas derrotas al partido oficial cuando éste se encontraba en el cenit de su poderío. No obstante, se ignoran los factores que dan sustento al surgimiento y la popularidad del Acción Nacional en Yucatán.

A nivel teórico existen dos explicaciones para analizar la fortaleza panista en Yucatán. Una explicación de corte cultural fundamenta la reacción yucateca propanista en la singularidad de sus habitantes, incluyendo sus sentimientos de alienación del resto de México y del gobierno central priista. Otra posición teórica de tipo utilitarista indica que los yucatecos podrían favorecer al PAN motivados por cálculos de tipo económico personales y de evaluaciones de las condiciones económicas en general. En otras palabras, votan por este partido porque se benefician de él.

Dentro de las limitaciones de los datos estadísticos disponibles, este trabajo trata de solventar la brecha existente entre el desempeño electoral panista - a nivel descriptivo- y las explicaciones desarrolladas a nivel teórico para examinar empíricamente los factores que podrían sustentar el crecimiento histórico del PAN en el estado del sureste mexicano. En específico, se intenta discernir si las preferencias de los yucatecos por el PAN se basan en las orientaciones culturales tradicionales de la identidad yucateca, expresadas en altos niveles de regionalismo, etnocentrismo e internacionalismo, ${ }^{2}$ o si se deben a preferencias políticas económicas de corte neoliberal. De particular interés será determinar si la importancia de estas orientaciones varía por nivel educativo, con los mejores educados estando menos restringidos por la cultura yucateca, y los menos educados mostrando mayor predisposición a apoyar un partido de derecha debido a sus orientaciones culturales tradicionales. 
En tanto que ambas posturas teóricas serán analizadas empíricamente en secciones posteriores, es necesario clarificar primero dos conceptos fundamentales que sustentan este artículo: Yucatán como región e identidad regional yucateca. Es evidente que el estudio de la "región" presenta complicaciones de orden metodológico. El término puede estar sujeto a interpretaciones, múltiples conceptualizaciones y dificultades asociadas a su uso como unidad de análisis, que pueden terminar en una "irresolución" de la región, como afirma Bunce (2001). ${ }^{3}$ La numerosa bibliografía sobre las regiones mexicanas (Carrillo, 1972; Barkin y King, 1970; Bassols, 1979, Klesner, 2001a) atestigua la dificultad de agrupar entidades federativas en diferentes categorías mediante criterios geográficos, políticos, económicos, sociales o culturales. ${ }^{4}$

Para evitar caer en la irresolución de la que habla Bunce, conceptualizo como "identidad regional yucateca" la identidad básica que los individuos que habitan en el estado de Yucatán han adquirido a través de las distintas experiencias históricas y culturales que los diferencian de otras regiones, y cuyas ataduras a la región se extienden más allá de las instituciones gubernamentales locales o nacionales. Para el término "región yucateca" retomo elementos de Markusen (1987) y la defino como la sociedad territorial contigua que posee un medio ambiente físico, político, socioeconómico y cultural, en una estructura espacial distinta de otras regiones y de otras unidades territoriales, locales, estatales o nacionales. Por tanto, las expresiones "yucateco" y "regionalismo yucateco" se referirán de manera exclusiva a los habitantes del estado de Yucatán y a su espacio geográfico actual, y no a todos los pobladores o al espacio geográfico que conforman los tres estados de la península yucateca (Quintana Roo, Campeche y Yucatán).

Una vez hechas estas precisiones, se prosigue con una breve revisión de la fortaleza electoral histórica del PAN en el estado de Yucatán, para después atar la discusión a la literatura sobre regionalismo y comportamiento político a nivel nacional y yucateco.

\section{La fortaleza electoral histórica del PAN en Yucatán}

En la era del sistema del partido único, nadie puede disputar que el PRI dominó la escena política yucateca durante 71 años. Similar a lo ocurrido en otras partes de México, el tricolor fortaleció la supremacía del Ejecutivo y cambió favores políticos y económicos por apoyo político (Rubio, 1998). Tales mecanismos se complementaron con el uso del aparato de Estado para coaccionar, cooptar o, en tiempos electorales, recurrir a fraude para asegurar la victoria en aquellas instancias donde el PRI era retado.

Pero una revisión más minuciosa de la historia electoral yucateca revela la existencia de una poderosa oposición en la forma del PAN. Esta oposición apareció por primera vez a mediados de la década de los cuarenta del siglo pasado, y se fortaleció a través de los años mediante la construcción de una base popular que le permitiría, eventualmente, retar al PRI en Mérida y, en una etapa posterior, en el interior del estado de Yucatán.

Entonces, una descripción cronológica del ascenso del PAN yucateco tendría que comenzar ineludiblemente en 1946. En el marco de una elección caracterizada por acusaciones de fraude serial, el candidato del partido blanquiazul gana el voto urbano meridano, pero termina perdiendo estrechamente la carrera por el congreso local cuando se contabilizan los votos rurales, cuya legitimidad, de acuerdo con Montalvo y Vallado (1997), se duda. 12 años después, la amenaza opositora se materializa. En un desenlace inesperado que resonó a nivel nacional y local, en 1958 un candidato panista es electo diputado federal por un distrito meridano predominantemente urbano, derrotando a su contrincante priista por casi seis mil votos (Montalvo y Vallado, 1997). El significado de esta elección no puede subestimarse dado que ocurre en la cúspide de la hegemonía priista, cuando los disentimientos de cualquier tipo, incluso los expresados en las urnas, eran limitados de manera severa por el aparato PRI/Gobierno. 
Pero la victoria emblemática panista ocurrió en la década de 1960. Los éxitos previos, además de demostrar que el partido oficial no era invencible, también constituyeron los cimientos de un voto duro asociado con el PAN yucateco, lo cual le permitiría enfrentar al PRI con posibilidades reales de triunfo como ocurrió en Mérida. Si durante el proceso evolutivo panista el PRI continúa imponiéndose en todas las elecciones federales, estatales y locales, para 1967 tal dominio tricolor es quebrantado por Víctor Manuel Correa Rachó (PAN), quien gana fácilmente la alcaldía de Mérida por un margen de tres a uno (Spalding, 2000). De esta manera, el PAN se convierte en partido de gobierno y catapulta dicha conquista como una de las más importantes a nivel nacional en su larga vida opositora. Asimismo, la victoria sin precedentes serviría a Correa como una plataforma para su posterior aventura: la elección para gobernador de Yucatán en 1969. Sustentado por acusaciones de corrupción y descontento popular contra la administración estatal priista, y "jugando con el regionalismo yucateco" en su campaña (Mabry, 1973: 82). Correa, según ciertos observadores, gana la gubernatura sólo para ser ilegítimamente privado de ella mediante un fraude priista de monumentales dimensiones (Mabry, 1973), y también bajo un clima político de represión e intimidación gubernamental encabezado por el PRI (Shirk, 2005; Montalvo y Vallado, 1997). ${ }^{5}$ El resultado de las elecciones, fraude o no, alarmó sobremanera a la cúpula nacional priista.

Sin embargo, y a pesar de tan disputada contienda electoral, el PAN yucateco entró en un período de latencia. Como forma de protesta por todo lo sucedido en 1969, el partido se abstuvo de participar en varios comicios. Pero a pesar de este autoimpuesto repliegue, el partido entró a lo que Spalding (2001) llama "la segunda fase del activismo panista" a finales de los años ochenta del siglo XX. A partir de esta fecha, la política yucateca sería sinónimo de PRI y PAN.

Un breve repaso al resurgimiento panista comenzaría con la exitosa campaña de Ana Rosa Payán Cervera para las elecciones a diputados en 1988. Dos años después, Payán Cervera, ya convertida en la más prominente panista yucateca, emerge victoriosa de una muy cerrada elección para convertirse en el segundo alcalde meridano emanado de este instituto político. A partir de este momento, el PAN mantuvo control sobre la capital yucateca ganando seis veces consecutivas las elecciones para alcalde (1990-2010), algunas de ellas por un gran margen de votos, y simultáneamente se expandió por todo el estado. Para principios de los noventa el enfrentamiento panista-priista se movió a una arena diferente: el Congreso yucateco. Con cada elección el PAN capturaría otra curul priista hasta eventualmente convertirse en mayoría legislativa. Entre tanto, el número creciente de seguidores en Mérida y en otros centros urbanos yucatecos, además de en varias comunidades rurales, auguraba una derrota priista tan inexorable como histórica en su último reducto: la gubernatura. Para 2001 el panista Patricio Patrón Laviada, un exalcalde meridano, era el nuevo encargado del Poder Ejecutivo estatal tras imponerse al priista Orlando Paredes Lara por un margen relativamente confortable. ${ }^{6}$

La preponderancia del PAN en tierras yucatecas nunca fue tan evidente como en la elección presidencial de 2006. La Coalición por el Bien de Todos (CBT), representada por Andrés Manuel López Obrador (AMLO), barrió el sur mexicano con excepción de Puebla y Yucatán. En Yucatán, el PAN, con Felipe Calderón Hinojosa, se impone a esta coalición por 239201 sufragios (IFE, 2006). ${ }^{7}$ Teniendo en cuenta todos los factores involucrados en una elección y los resultados específicos en cada entidad federativa, quizá resulta aventurado afirmar que la amplia derrota yucateca pudo haberle costado la presidencia a López Obrador, pero el conteo final de los votos nacionales revela una victoria panista sobre el candidato de la izquierda de apenas 243934 votos sobre 41791322 emitidos (IFE, 2006). ${ }^{8} \mathrm{Y}$, como se dijo, 239201 provienen de la diferencia aritmética de votos 
totales obtenidos por el PAN en Yucatán menos los conquistados por la CBT en esa entidad federativa. ${ }^{9}$

En suma, es claro que el PAN ha sido un actor histórico importante en el escenario de la política yucateca y que su presencia se remonta a la época cuando los partidos opositores enfrentaban grandes restricciones en su labor política, incluyendo actividades de corte proselitista. Los hechos también demuestran que el PAN ha construido de manera paulatina un voto duro y de carácter urbano, y que la dicotomía PRI-PAN ha dejado poco espacio para otras expresiones partidistas, en especial para aquellas ideológicamente asociadas con la izquierda. Aún más, se sabe que la oposición al PRI comienza en los estados mexicanos norteños y en Yucatán. En el caso del norte mexicano se puede hablar de democracia por contagio dada la cercanía con Estados Unidos. En Yucatán, como dice Spalding (2000: 4), la fortaleza electoral del PAN "no ha sido bien explicada todavía”. Entonces, iqué factores explican la popularidad del partido azul y blanco en Yucatán? ¿Cuáles son las causas del comportamiento electoral opositor de una gran porción de votantes yucatecos? ¿Existen efectos regionales en la política mexicana? Es claro que la literatura desarrollada a nivel agregado —nacional- aborda muchas de estas cuestiones, pero tales explicaciones no son compatibles con el caso yucateco, como se verá en la siguiente sección.

\section{Una breve revisión de la literatura sobre regionalismo y política electoral en México y Yucatán}

Las cuestiones expresadas en este artículo conciernen a la literatura sobre regionalismo y política electoral en México. Como se mencionó, la región se ha convertido, a nivel macro, en una de las variables cruciales en comportamiento y democratización (Klesner,1993, 1995, 200la, 2012; Baker, 2006; Hiskey y Bowler, 2005; HuescaDorantes, 2003; Cornelius, Eisenstadt y Hindley, 1999; Beer, 2003; Rodríguez y Ward, 1995), en particular tras la elección presidencial de 2006 que dio como resultado un país dividido entre estados azules (PAN) y amarillos (PRD) (Lawson 2006, 2007; Klesner, 2007). En específico, el PAN se convirtió en la mayor fuerza en el centro y norte del país así como en Yucatán. Por el contrario, la base electoral del PRD se encuentra en muchos de los estados del sur mexicano y en la Ciudad de México (Klesner, 2001b), mientras que el PRI todavía conserva una presencia nacional (Cady, 2003).

Esta brecha o hendidura regional de conducta electoral parece seguir patrones de desarrollo económico y niveles educativos regionales similares a los que la teoría de la modernidad predijo. Los primeros estudios de tipo mostraron que los segmentos urbanos, educados, y las clases media y alta, tendían a identificarse más con el derechista PAN. De igual manera, el apoyo hacia el PRI es alto entre los menos educados, de menor ingreso, y la población rural (Klesner, 1993). Entonces, las regiones con mayor concentración de clases media y opulenta son más propensas a votar por el PAN; por el contrario, las regiones con mayor concentración de habitantes de menores ingresos son más propensas a ser perredistas o priistas (Camp, 1999). Como se sabe, el norte es la región mexicana más desarrollada, en tanto que el sur es fundamentalmente de ingreso bajo, rural, agrario e indígena. ${ }^{10}$ Entonces, las diferencias regionales en fortaleza partidista reflejan las diferentes condiciones económicas. Sin embargo, Yucatán es la excepción a este patrón dado que es un estado relativamente empobrecido localizado en el sureste mexicano, pero donde el PAN es el principal partido opositor en lugar del PRD como se pensaría.

La segunda línea teórica relaciona el patrón regional de voto y la fortaleza partidista con los cambios económicos derivados de las políticas neoliberales (Klesner, 200la). Desde esta perspectiva, el proneoliberal PAN es más fuerte en las áreas más beneficiadas por el libre comercio, como el norte, en tanto que el nacionalista PRD, de centro-izquierda, obtiene apoyo en las áreas menos favorecidas por la apertura económica neoliberal, 
como el sur. En el caso de la Ciudad de México, la dominación electoral perredista puede explicarse por las tradicionales orientaciones nacionalistas, así como por el impacto negativo del neoliberalismo en ciertas industrias nacionales, incluyendo paraestatales, que prosperaron bajo el modelo de sustitución de importaciones pero que no pudieron competir contra corporaciones transnacionales.

Un tercer enfoque explica las conductas electorales relacionándolas con escisiones o divisiones religiosas. El conservador PAN tiende a ser más popular en regiones donde la Iglesia católica ha mantenido una férrea influencia, como es el caso de la región central, algunas ciudades del norte y Yucatán. Sin embargo, son menos claros los efectos de la religión sobre el apoyo electoral al PRD y al PRI. Ambos partidos son más liberales en causas sociales como derechos, aborto o legalización del matrimonio entre homosexuales, y claramente son más seculares que el PAN. Pero, quizá con la excepción del cosmopolita y liberal Distrito Federal, no se puede argumentar de manera convincente que el PRD es más popular en el sur porque esta región es menos católica que otras. De hecho, se puede argüir que algunos estados del sur son más tradicionales que otras entidades federativas mexicanas de cualquier región.

Pero, con todos sus méritos, estos enfoques desarrollados a nivel macro no exactamente capturan lo que son los "efectos regionales" en conducta electoral (Baker, 2006). Por ejemplo, Baker pregunta cómo se puede explicar que "individuos con características demográficas iguales pero diferente situación geográfica regional tienen vastas probabilidades de votar de manera diferente por determinado candidato."ll Una posible explicación radica, según Baker, en el contenido de la discusión política que se da en las regiones mexicanas. Otra posibilidad es que existen diferencias específicas inherentes a cada región en cultura política, como se argumenta en este artículo.

Resumiendo, la literatura sobre regionalismo y conducta electoral en México desde una perspectiva a nivel macro, en tanto que es teóricamente valiosa, aún no explica suficientemente dos cuestiones fundamentales: por qué algunos partidos opositores son muy fuertes en algunas regiones pero no en otras, y qué constituye el "efecto regional". Por eso, la cuestión esencial de esta investigación es examinar si las orientaciones culturales tradicionales de una identidad local ayudan a explicar el apoyo electoral al PAN en Yucatán.

A la fecha, la muy limitada investigación en conducta electoral yucateca se ha enfocado en explicar las raíces históricas y la composición demográfica del apoyo al PAN. En esta literatura se hacen hipótesis sobre la "singularidad del regionalismo yucateco", como Alberro (1994) nota, una variable fundamental para entender el apoyo ciudadano hacia el PAN. Más aún, otros estudios sugieren que la oposición al PRI emergió como una consecuencia indirecta de las reformas estructurales implementadas en los años ochenta. Ninguna de estas hipótesis ha sido empíricamente puesta a prueba.

Entonces, en el caso de Yucatán, la escasa producción académica puede ser, a grandes rasgos, categorizada en dos vertientes: una que ve el voto a favor del PAN como consecuencia de las reformas neoliberales, y la otra que percibe este apoyo como una reacción a la hegemonía del gobierno central, alimentada por agentes locales. Entre la primera vertiente de tipo económico-utilitarista se encuentra Spalding $(1999,2000)$. Esta politóloga argumenta que el cambio a una economía de mercado más liberal debilitó la maquinaria corporativista priista, y la liquidación de CORDEMEX ${ }^{12}$ dañó en grado superlativo a los trabajadores urbanos de bajo ingreso así como a los rurales. Consecuentemente, el PAN pudo convertir en ganancias partidistas - electoralesla molestia de estos grupos demográficos que se desprendió del PRI. ${ }^{13}$

Otros científicos sociales (Canto, 2001; Sierra, 1995; Needler, 1995; Alisky, 1980) creen que el voto panista yucateco es una forma de protesta y una reacción a la hegemonía del centro y del gobierno central. Esta hostilidad cultural es también propagada por 
actores muy influyentes, como la Iglesia católica y el conservador y propagandístico Diario de Yucatán, que contribuyen a inclinar el voto yucateco hacia el PAN, especialmente entre las clases urbanas (Spalding, 1999, 2000, 2001; Montalvo y Vallado, 1997; Sierra, Paz y Huchim, 1986; Sierra, 2000).

Una vez descritas las dos vertientes teóricas, es necesario analizar la relación entre identidad local y voto panista, basada, como ya se dijo, en una reacción hacia el centro o en la forja de actitudes económicas favorables a una economía de mercado más liberal. La siguiente sección se encarga de ello.

\section{Datos y cuestiones empíricas}

Para examinar las cuestiones señaladas siguiendo una metodología cuantitativa, se utiliza una encuesta realizada en el estado de Yucatán y financiada por la Universidad de Kentucky que incluye los indicadores a usarse en esta investigación — de ahora en adelante referida como Encuesta Yucatán-. El estudio de opinión pública se efectuó en Mérida y en dos pueblos, Hunucmá y Tixkokob, ambos localizados en un radio de 25 kilómetros de la capital yucateca, en marzo de 2005. Se eligieron aleatoriamente 400 individuos, de 18 años o más, a los que se entrevistó cara a cara. El cuestionario fue diseñado y revisado por el autor de este artículo, pero una firma local — Servicios Peninsulares de Mercadotecnia - llevó a cabo el estudio.. ${ }^{14}$

El concepto fundamental de la explicación teóricocultural es el de identidad yucateca. Se argumenta que las experiencias históricas del estado de Yucatán y de la región que lo rodea fueron distintas a las de otras regiones mexicanas. ${ }^{15}$ En consecuencia, se desarrollaron orientaciones culturales e identidades diferentes a las del resto de México y, con ello, surgió una identidad yucateca parcialmente constituida por cuatro orientaciones culturales: 1) una primordial identificación con la región yucateca, la cual incluye un sentimiento alto de apego a lo subnacional versus nación- estado, o hacia la matria sobre la patria; 2) orientaciones políticas conservadoras; 3) un etnocentrismo cultural que exalta el legado maya de la región sobre la herencia nacional azteca que el estado posrevolucionario priista idealizó y exaltó; y 4) actitudes favorables a Estados Unidos o internacionalismo que contrasta con los sentimientos de xenofobia y desconfianza que el PRI promovió durante su amplio control del sistema político mexicano de 1929 a 2000. ${ }^{16}$ Siguiendo este argumento, la reacción yucateca de rechazo al centro y su oposición histórica al PRI, percibido este partido como agente de la hegemonía del gobierno central mexicano, podrían tener bases culturales relacionadas con la identidad yucateca y a su vez podrían explicar por qué la popularidad del derechista PAN surge y permanece relativamente alta en el estado de Yucatán.

Con base en lo anterior, se desarrollan y se examinan dos diferentes modelos que vincularían la identidad regional de los yucatecos con el apoyo al PAN. El primero, denominado el "modelo de atención cultural" de lealtad panista, asume que hay un efecto directo entre la identidad yucateca y el apoyo al PAN, sin que intervenga o medie una determinada posición neoliberal en temas de políticas (policy). Específicamente, este modelo asume que los yucatecos con una identidad regional acendrada apoyan al PAN, no por su conservadurismo o posición ideológica en favor del neoliberalismo, sino por su defensa de la región y por observar amenazas, ficticias o no, de la hegemonía del PRI y del centro hacia su identidad cultural. En este modelo, un voto por el PAN es la mejor defensa para la amenaza externa del centro. De igual manera, el modelo asume que los líderes panistas articulan estas preocupaciones inherentes a la identidad yucateca como discurso propio.

El segundo modelo, el cual denomino "modelo de efectos de política pública" (policy), asume que las orientaciones culturales vinculadas con la identidad yucateca influyen de manera indirecta en el apoyo electoral al PAN. Estas orientaciones conducen a actitudes favorables hacia reformas neoliberales que, 
en cambio, resultan en un apoyo mayor al derechista PAN. La lógica es ésta: se conjetura que la cultura regional, como se ha examinado en este estudio, está enraizada en un sentimiento histórico de desconfianza hacia el gobierno central, pero sí favorable al mundo exterior, incluyendo a Estados Unidos. Entonces, los individuos que abrazan esta cultura deben estar predispuestos a apoyar las políticas económicas neoliberales que reducen el rol del Estado como motor de desarrollo económico, que apuestan por el libre mercado, y que buscan fortalecer la integración con Estados Unidos. En consecuencia, un mayor apoyo a las reformas neoliberales conducirá a un apoyo mayor al derechista PAN, partido que históricamente ha articulado tal filosofía económica. Este modelo se confirma principalmente entre los yucatecos de mayor educación, dado que estarán, a diferencia de los menos educados, muy pendientes de la política económica relacionada con el programa neoliberal y sus consecuencias. ${ }^{17}$

\section{Análisis estadístico}

Antes de evaluar los modelos culturales y de política de apoyo electoral al PAN mediante la técnica de regresión lineal, es imperativo señalar cómo se creó la variable dependiente "apoyo electoral al PAN", así como las otras variables utilizadas. La Encuesta Yucatán incluyó dos indicadores para medir intención de voto o voto actual en una elección específica, pero como frecuentemente sucede en México — en especial durante periodos de poca actividad electoral- ${ }^{18}$ estos indicadores arrojaron un gran número de respuestas indeterminadas - o sea, indecisos- y también de datos faltantes. ${ }^{19}$ Entonces, para evaluar el apoyo electoral al PAN se tomó la decisión de usar un indicador sucedáneo para evitar las respuestas indeterminadas o missing data. El indicador sucedáneo es "lealtad partidista", el cual se funda en una escala construida a partir de los dos indicadores siguientes:
1) la opinión que tiene el encuestado sobre el PAN, y 2) su grado de simpatía hacia este partido. ${ }^{20}$ Por tanto, quizá sea mejor describir "lealtad partidista" como indicador de un posible apoyo electoral a cualquier partido político.

En el análisis estadístico, se utilizan tres variables culturales — regionalismo, actitudes hacia Estados Unidos y etnocentrismo - en el análisis estadístico como indicadores de la identidad yucateca. Puesto que la base de datos no incluye indicadores para el cuarto componente teórico — conservadurista—, éste no será puesto a prueba. Las tres variables señaladas líneas arriba fueron construidas mediante escalas factoriales de una batería de 12 preguntas, de las cuales cinco cargaron en tres dimensiones diferentes (Eigenvalue $>$ ). ${ }^{21}$ Para medir actitudes hacia el neoliberalismo se utilizan dos variables como indicadores de dos componentes fundamentales del modelo neoliberal en México: actitudes hacia el Tratado de Libre Comercio de América del Norte (TLCAN) y actitudes sobre privatizaciones. ${ }^{22}$ Cinco reactivos fueron utilizados para construir las dos dimensiones mencionadas (Eigenvalues〉1). El desarrollo de estos indicadores se explica con mayor detalle en el anexo $1 .{ }^{23}$ En este tenor, si bien es cierto que existen limitaciones en los datos estadísticos disponibles, es verdad también que las orientaciones culturales inherentes a una identidad yucateca y su relación con el PAN pueden ser evaluadas usando las variables descritas en forma cuantitativa.

La primera tarea en el análisis estadístico consiste en examinar el modelo de atención cultural de lealtad panista. Si el modelo es consistente con los datos estadísticos, debemos encontrar coeficientes de regresión estadísticamente significativos entre orientaciones culturales y apoyo al PAN cuando investigamos actitudes hacia la política económica actual. Esto indicaría que, independientemente de las actitudes que tengan los encuestados hacia el TLCANy las privatizaciones, aquellos con fuertes orientaciones culturales regionales son más propensos a favorecer al PAN. 
Para este propósito, el método estadístico usado es la regresión $\mathrm{MCO}$ - mínimos cuadrados ordinarios —, 24 donde las variables independientes son los componentes de la identidad yucateca - regionalista, etnocentrista e internacionalista, además de actitudes sobre el TLCAN y las privatizaciones-. Se incluyen actitudes sobre políticas públicas (policy) en las regresiones para examinar el impacto de las variables culturales cuando las actitudes hacia las políticas públicas son controladas (ver tabla 1). También se incluyen correlaciones de orden cero para contrastarlas con los coeficientes de la regresión que han sido ajustados por las variables de control. El objetivo primario es determinar si las orientaciones culturales yucatecas influyen directamente en el apoyo electoral al PAN, sin mediar en esta relación actitudes hacia las reformas neoliberales. Aún más, para evaluar si el PAN, como dice Spalding (1999: 17), tiende a contar con el apoyo de los segmentos demográficos de la "clase media, educados, y del sector profesional" ${ }^{25}$ la muestra se divide en tres estratos educativos. $^{26}$

La tabla 1 presenta los resultados del modelo de atención cultural. Como se puede observar en los coeficientes beta de la muestra total (ver tabla l, primera columna), donde se valoran los efectos directos de las variables independientes sobre la variable dependiente, manteniendo constantes las actitudes hacia las políticas públicas, hay poca evidencia que sustente el modelo de conciencia cultural en la Encuesta Yucatán 2005. Ninguna de las tres variables culturales es un predictor estadísticamente significativo de lealtad partidista hacia el PAN, ya sea para la muestra total o para los tres estratos educativos, cuando las actitudes hacia las políticas se mantienen constantes. Las correlaciones de orden cero para la muestra total y los estratos analizados también son débiles.

En forma sucinta, tres factores podrían explicar la carencia de evidencia empírica para sustentar el modelo de atención cultural de lealtad panista. Primero, el PRI no es más el partido presidencial y el símbolo de resistencia hacia el centro. En segundo lugar, algunas campañas panistas llevaban una gran connotación regional, pero el avance electoral de este partido quizá implicó que la carta regionalista local haya sido reemplazada por temas nacionales e internacionales de preocupación inmediata para el electorado. En otras palabras, el PAN ya se habría enfocado en otros temas antes que a manipular la aversión hacia el gobierno central encabezada por su propio partido. Finalmente, además de estos factores, puede ser relevante preguntarse si el número reducido de casos en el estrato de los menos educados ( $\mathrm{n}=128$ ) incidió en la significancia estadística de los componentes regionalistas y etnocentristas. ${ }^{27}$

El análisis estadístico ahora se enfoca a analizar el modelo de efectos de política pública de lealtad hacia el PAN. La hipótesis básica es que las orientaciones culturales de la identidad yucateca están asociadas con el apoyo a las reformas neoliberales que conllevan a una lealtad al PAN. La suposición subyacente a este modelo es que las orientaciones culturales tienen un efecto indirecto en la lealtad hacia el PAN. Por eso, la hipótesis contiene dos nexos: uno, la relación entre orientaciones culturales y apoyo a las reformas neoliberales; y dos, la relación entre apoyo a las reformas neoliberales y lealtad al PAN. Si hay un efecto indirecto, esperaríamos que ambas relaciones sean estadísticamente significativas. Con este fin, el análisis se efectúa para la muestra total y los tres estratos educacionales descritos en el modelo anterior, usando como indicadores actitudes hacia el TLCAN y privatizaciones.

Para analizar el modelo, se examina el segundo nexo: relaciones entre apoyo a las reformas neoliberales y lealtad hacia el PAN (ver tabla 2). Después, el análisis se dirige al anterior eslabón causal: relaciones entre orientaciones culturales y apoyo a las reformas neoliberales por estrato educativo. ${ }^{28}$

Entonces, al analizar primero la muestra total, es evidente que las actitudes hacia el TLCAN son el predictor más fuerte de lealtad panista (beta $=.185$ ) y el estadísticamente significativo al nivel .05 y superior. 
Las actitudes hacia las privatizaciones, por otro lado, contribuyen modestamente al modelo a juzgar por sus bajos coeficientes beta (beta $=.071$ ). Por segmento educativo, el nivel bajo presenta un fuerte nexo entre TLCAN y lealtad hacia el PAN (ver tabla 2, columna 4), pero no así entre privatizaciones y ese partido (beta =-.015). Paradójicamente, cuando la comparación se realiza con el estrato de bajo nivel educativo, las actitudes hacia las privatizaciones son más fuertes entre los encuestados pertenecientes al segundo estrato o medio, pero sus actitudes hacia el TLCAN son más débiles (ver tabla 2, columna 7). No sorprende entonces que la misma tendencia se vea en las correlaciones de orden cero para dicho subgrupo - .194 para el TLCAN, .171 para privatizaciones-. Para el estrato de nivel superior, los datos estadísticos muestran de forma clara cómo las actitudes hacia las políticas públicas se conectan con la lealtad hacia el PAN. Los coeficientes beta para las variables de tipo policy hacia el TLCAN (beta $=.255)$ y las privatizaciones (beta =.208) son las más fuertes de los tres grupos educativos analizados y las actitudes hacia el TLCAN son estadísticamente significativas al nivel .05 (ver tabla 2, columna 10).

Estos resultados sugieren que, cuando los niveles educativos de los entrevistados aumentan, éstos son más propensos a vincular ambas políticas - TLCAN y privatizaciones - con el apoyo partidista. En otras palabras, los mejores educados tienden a ser votantes más orientados a las políticas públicas mencionadas que los de bajo nivel educativo. Estos resultados confirman que una orientación favorable hacia las reformas neoliberales de libre comercio y privatización se enlaza con lealtad partidista hacia el PAN en mayor medida entre los más educados que en el caso de quienes cuentan con educación media y baja. Sin embargo, también es notable la influencia del TLCAN en la formación de lealtad panista entre aquellos de menor logro educativo.

El análisis ahora se concentra en el primer eslabón del modelo: la relación entre los componentes culturales de la identidad yucateca y las actitudes hacia las reformas neoliberales (TLCAN y privatizaciones) entre diferentes subgrupos mediante la regresión MCO, como se ve en la tabla 3. Las regresiones introducen también controles para nivel de ingreso y tipos de empleo.

En general, el análisis estadístico provee evidencia substancial para sustentar la hipótesis de que las orientaciones culturales influyen sobre las actitudes hacia el TLCAN a través de los diferentes niveles educativos mientras que, al mismo tiempo, muestra poca evidencia de los efectos hipotéticos de las orientaciones culturales en el componente "privatizaciones". Dos estratos en particular sobresalen por la fortaleza de sus asociaciones entre las variables culturales y el TLCAN: los de bajo y los de alto nivel educativo. ${ }^{29}$ Es interesante notar que, mientras entre los menos educados es el componente regionalista el que presenta mayor influencia en las actitudes hacia el TLCAN, para los de mayor educación es la actitud hacia Estados Unidos la variable de mayor influencia en sus orientaciones. Este hallazgo sugiere que los más educados podrían estar perdiendo sus ataduras locales o regionales y estar orientándose al plano internacional, en contraste con los de menor nivel educativo que sí están atados a la región o localidad.

Con respecto a las actitudes hacia las privatizaciones, esta variable vincula solamente el componente etnocentrista entre aquellos con educación media, y con actitudes hacia Estados Unidos entre el grupo de alto nivel educacional (ver tabla 3, columnas 10 y 16). La variable "identidad regional" no tiene relevancia en la formación de actitudes hacia las privatizaciones de cualquier estrato. Aún más, no hay asociaciones entre las tres variables culturales de la identidad yucateca y privatizaciones entre los de baja educación: sólo las actitudes hacia Estados Unidos están vinculadas, y en forma débil, con dicho componente del programa neoliberal, como puede verse en su correlación de orden cero (.123). Por tanto, las orientaciones culturales importan más en la formación de actitudes hacia el 
TLCAN que hacia las privatizaciones, lo que quizá refleja la importancia sumaria del TLCAN entre los encuestados.

\section{Conclusión}

La actual literatura en conducta electoral en México ha mostrado muchos factores que impactan al votante mexicano, incluyendo el componente "región", pero sin precisar cómo esta última variable moldea la conducta electoral del votante y qué mecanismos se involucran en el concepto. Este trabajo examina empíricamente un mecanismo tal, el rol de la cultura regional en promover el voto de oposición hacia el PAN.

Con los datos disponibles, dos modelos se proponen y analizan: el modelo de atención cultural de lealtad panista y el modelo de política pública. Como se indicó, el análisis estadístico revela poca evidencia para el modelo de atención cultural, pero sí un apoyo considerable al modelo de efectos de política pública hacia el PAN, en especial entre aquellos de mayor nivel educativo.

Por tanto, la tradicional cultura local aparece como factor para explicar por qué los yucatecos, aun los menos favorecidos económicamente, apoyan al conservador PAN. Factores de tipo económico pueden ser igual de importantes entre las clases altas, pero este análisis apoya el argumento elaborado por aquellos politólogos que desde hace mucho tiempo han clamado que la cultura regional de los habitantes de Yucatán importa para explicar el apoyo popular hacia el PAN en esa entidad federativa. Además, los resultados podrían sugerir, aunque de manera tentativa, que un partido considerado de izquierda o con políticas económicas proestatistas, antineoliberales y con un discurso antiglobalizador no encontraría gran eco entre el votante típico yucateco. En suma, el electorado yucateco se desplaza en el espectro ideológico hacia el sector de centro-derecha por cuestiones inherentes a su cultura local. Si estas cuestiones pueden ser alteradas por un candidato izquierdista carismático, o por saturación del votante yucateco ante la dicotomía PAN-PRI, o por cualquier factor de otra índole, está por verse todavía. Los resultados de esta investigación sugieren que no.

\section{Anexo 1: escalas e indicadores}

Componentes de la identidadyucateca: Tres escalas factoriales —etnocentrista, regionalista e internacionalista - se crearon usando cinco ítems (Eigenvaluesl). Componente etnocentrista. Dosítems: 1) basado en lo que usted sabe, la civilización azteca fue más importante que la maya; 2) los mexicanos —del resto del país- son más sinceros y honestos que los yucatecos. Componente regionalista. Dos ítems: 3) si usted pudiese elegir, ¿cómo preferiría ser llamado: yucateco o mexicano?; 4) Si Yucatán fuese un país, ¿sería más rico que como es ahora, un estado de la República mexicana? Componente internacionalista. Un reactivo: 5) ¿cuál es su opinión de los Estados Unidos?

Componentes de actitudes hacia el neoliberalismo: dos escalas factoriales - actitudes hacia el neoliberalismo y actitudes hacia las privatizaciones - fueron creadas usando cinco reactivos (Eigenvalue>1). Componente TLCAN. Tres ítems: 1) opinión general sobre el TLCAN; 2) iusted cree que el TLCAN ha sido (muy malo a muy bueno) para México?; 3) ia usted le gusta o no que en Yucatán uno pueda comprar mercancía extranjera de todo tipo (electrónica, ropa, discos, zapatos, etcétera) que antes del TLCAN no estaba disponible? Componente privatizaciones. Dos ítems: 4) ila venta de empresas del gobierno como bancos, teléfonos, líneas aéreas entre otras ha sido (muy negativa a muy positiva) para gente como usted?; 5) basado en lo que sabe, iqué le parece a usted la venta y liquidación de CORDEMEX?

Lealtad partidista: Lealtad al PAN. Dos items: 1) opinión general del PAN; 2) ¿Cuánto simpatiza con el PAN? Lealtad al PAN (correlación de Pearson entre los reactivos 1 y 2 es .672; Alpha-Cronbach .804). Ambos ítems fueron sumados y divididos entre dos, y 
codificados bajo el siguiente criterio: 1-2.5 baja lealtad; 3 media lealtad; 3.5 a 5 alta lealtad.

\section{Notas}

${ }^{1}$ Traducción del autor.

${ }^{2}$ Véase Figueroa $(2009,2013)$ para una descripción más detallada de estos componentes.

${ }^{3}$ Este argumento coincide con el de Ross (1997: 4344) cuando habla de las dificultades que se presentan cuando la "cultura" es el elemento central de un estudio.

${ }^{4}$ Esta breve discusión da pie a una plétora de preguntas: ¿Qué es una región? ¿Dónde comienza una y termina otra? ¿Los atributos y singularidades de una región son exclusivos de ella? En el caso de México, ipueden ignorarse diferencias interregionales en estados como Veracruz y Quintana Roo? ¿Existe un norte o hay varios? ¿A qué región pertenecen estados que pueden fácilmente ser parte de dos o más como Puebla, Morelos, Zacatecas, San Luis Potosí? ¿Cuántas regiones hay en México? ¿Bajo qué criterios deben agruparse los estados mexicanos en bloques regionales?

${ }^{5}$ Debe tenerse en cuenta que, en este periodo particular, la hegemonía priista sólo se veía amenazada en Sonora y Baja California, dos estados norteños localizados al otro extremo de la República mexicana.

${ }^{6}$ Debe recalcarse que el PAN no pudo mantenerse en la gubernatura yucateca tras el sexenio (2001-2007) de su militante Patricio Patrón Laviada. En unos comicios caracterizados por su alta participación, los yucatecos eligen a Ivonne Ortega Pacheco, la abanderada prisita, como titular del Poder Ejecutivo para el periodo 20072012. Esteinesperadoresultadoquizá seala consecuencia de una combinación de factores que se confabularon en contra del partido azul y blanco. En primer término, el PAN sufrió un tormentoso proceso interno de selección de candidato que culminó con la renuncia de Ana Rosa Payán Cervera, la panista más connotada. En segundo lugar, a pesar de sus diferencias ideológicas, el PRI y el PRD forjaron una alianza electoral. Como tercer factor deben señalarse las campañas y los candidatos. La campaña panista nunca permeó entre la población, y mientras que el PRI enfatizó la juventud y vitalidad de Ortega Pacheco (37 años), el candidato panista Abreu Sierra (57 años) proyectaba una imagen física endeble, casi senil, que contrastaba de manera notoria con la de Ortega Pacheco. Finalmente, Patricio Patrón Laviada fue acusado de corrupción en los últimos meses de su mandato. También es importante notar que el PRI obtuvo la victoria en la elección para gobernador más reciente (2012), y que pierde la contienda por la alcaldía meridana a manos del PAN. En general, se mantiene el bipartidismo PAN-PRI en la política yucateca.

${ }^{7}$ Los resultados de la elección presidencial de 2006 en el estado de Yucatán fueron los siguientes: PAN, 364353 votos, equivalentes a $46.17 \%$ del total estatal; Alianza por México, 260116 votos (32.9\%); CBT, 125 152. El 15.86\% de los votos totales (IFE, 2006). Nótese la diferencia de 239201 votos en favor del abanderado panista Felipe Calderón Hinojosa sobre el candidato por la CBT, Andrés Manuel López Obrador.

${ }^{8} \mathrm{O}$ sea, restando los votos yucatecos, Calderón Hinojosa supera a AMLO por apenas 4733 votos. Los resultados dela elección presidencial del 2006 fueron los siguientes: PAN, 15000284 votos, equivalente al 35.89\% del voto total nacional; CBT, 14756350 votos (35.325); Alianza por México, 9301441 votos, es decir, 22.26\% de los votos totales (IFE, 2006).

${ }^{9}$ Quizá el argumento anterior, esgrimido más para subrayar la fortaleza electoral del panismo yucateco que para ilustrar el bajo desempeño de AMLO en este estado, pueda contextualizarse mejor si consideramos que, a nivel teórico, los clivajes particulares de Yucatán - estado relativamente pobre, ubicado en el sur de México, gran porcentaje de población indígena y rural- predecían una victoria de la izquierda con AMLO, y no un triunfo del partido presidencial en turno. Es importante mencionar que en 2006 la popularidad del presidente Vicente Fox y la del propio gobernador panista Patrón Laviada iban a la baja. Tal 
tendencia suponía que el votante yucateco iba a castigar electoralmente al PAN, pero, como vimos, no ocurrió así

${ }^{10}$ Los estados con mayor porcentaje de población indígena tienden a ser menos democráticos, según Hernández (2000).Tal hecho no es solamente una cuestión de raza, sino un reflejo de la subyugación histórica de estas etnias a grupos políticos locales o caciques.

${ }^{11}$ Traducción del autor. Baker (2006: 4-5) extiende tal argumento mediante la siguiente comparación. Dos individuos de clase alta, con iguales niveles educativos, católicos, liberales en ideología económica, pero con una diferencia crítica: uno vive en una ciudad del norte mexicano y el otro en una ciudad del sur. La probabilidad de que el norteño vote por el PAN es mayor que la de su compatriota sureño.

${ }^{12}$ CORDEMEX fue una empresa paraestatal yucateca que heredó los remanentes de la otrora poderosa industria privada henequenera de finales del siglo XIX y principios del XX. La liquidación de CORDEMEX en 1991 impactó a los yucatecos porque, a pesar de las acusaciones de corrupción que pesaban sobre ella y su gran ineficiencia, funcionaba en la práctica como un sistema de bienestar social (Baklanoff, 2008: 7). El ítem CORDEMEX, en esta investigación, funciona como proxi para construir la variable "privatizaciones".

${ }^{13}$ Este argumento, además de que no se ha probado de manera empírica, tampoco puede explicar las victorias panistas previas al advenimiento del modelo neoliberal.

14 Entiendo y comparto la preocupación expresada por un dictaminador anónimo cuando sugiere cierta cautela en extrapolar los resultados de la Encuesta Yucatán -efectuada en un área geográficamente reducida a Mérida y dos poblaciones aledañas-. Sobre este punto, la investigación sobre actitudes políticas en Yucatán diferencia muy poco entre lo local - Mériday lo estatal -Yucatán-. En otras palabras, el uso de Yucatán hace referencia a todo lo que ocurre en el estado, indistintamente de si Mérida es en realidad el centro de la investigación, y de si las conclusiones se derivan de lo que acontece en esa localidad solamente.
Este tratamiento puede estar fundado en la importancia de Mérida como capital y porque, a fin de cuentas, el surgimiento del PAN como oposición real se da en esta localidad y después se expande hacia el interior del estado. Opto por continuar tal tendencia basado en la disponibilidad de los datos estadísticos, a pesar de la inquietud que existe sobre este tratamiento, y dejar para una investigación futura una encuesta a gran escala para todos los distritos electorales yucatecos. En este artículo, Mérida y su hinterland - Hunucmá y Tixkokob- abarcan tres de los cinco distritos federales electorales yucatecos. Quiero agradecer los comentarios de este dictaminador anónimo.

15 Existe un gran número de autores que enfatizan tal argumento, desde el clásico Yucatan: a worldapart, editado por Moseley y Terry en 1980, The Machete and the Cross, publicado en 1997 por Dumond, hasta Figueroa (2009, 2013).

16 Figueroa (2013) ha probado empíricamente la existencia de tres - regionalismo, etnocentrismo e internacionalismo- de los cuatro componentes de la identidad de los habitantes del estado de Yucatán. Entre las conclusiones más importantes destacan la preferencia yucateca por la matria, o ámbito local, que sobre la patria o ámbito nacional, y que tal preferencia es más alta en Yucatán que en otras regiones de México, Además, los yucatecos exhiben altos niveles de etnocentrismo, aunque varían según el estrato socioeconómico. Finalmente, no parece haber un gran cambiogeneracional de los niveles deidentidad yucateca entre cohortes jóvenes y de más edad. Para llegar a estos resultados se utilizaron una encuesta de opinión pública y análisis estadísticos, como distribuciones de frecuencias, análisis factorial y análisis de varianza.

${ }^{17}$ Estos supuestos teóricos no ignoran los arquetipos existentes en los partidos PRI y PAN en relación con sus programas económicos. En efecto, el Gobierno encabezado por el PRI implementó desde mediados de los ochenta el paquete neoliberal, y la distinción PAN-PRI, desde una comparación de modelos de 
desarrollo económico, puede ser un tanto nebulosa, especialmente de 1990 a la fecha. Pero también es verdad, y se ha documentado en extenso (Camp, 2007; Ard, 2003; Cansino, 2000; Casar y Peres, 1998; Mabry, 1973; Shirk, 2005, entre otros) que la postura ideológica tradicional del derechista PAN, además de estar basada en la doctrina de la Iglesia católica, se fundamenta históricamente en el liberalismo económico —con piezas clave como el libre comercio y el rol reducido del Estado-. Por el contrario, el PRI ha modificado su doctrina económica acorde con el presidente prísta en turno,y ha transitado a lo largo del siglo XX de un modelo de economía más cerrada — como el modelo de Sustitución de Importaciones - hacia otro de desarrollo hacia afuera, como el programa neoliberal. Como dice Otero (2004: 1-17), de los tres partidos mexicanos importantes, el PAN ha sido el más firme defensor del neoliberalismo, y también el garante de la continuidad de este modelo neoliberal tras la victoria de Vicente Fox. En otras palabras, existen sustentos teóricos firmes cuando se afirma que la probabilidad de que un mexicano con tendencias neoliberales se identifique y vote por el PAN es mucho mayor a que dirija tal sufragio o se alinee hacia el izquierdista y antineoliberal PRD o al camaleónico PRI.

${ }^{18}$ Debe recordarse que la Encuesta Yucatán se llevó a cabo meses antes de las elección presidencial de 2006.

${ }_{19}$ Por ejemplo, solamente 43 de los 400 entrevistados $(11 \%)$ respondió la pregunta de por cuál partido iban a votar en la elección presidencial de 2006.

20 Los dos indicadores mencionados arriba están estrechamente relacionados con la intención de voto que los encuestados tenían para la elección presidencial de 2006 - la correlación de Pearson entre intención de voto para el PAN en 2006 y lealtad panista es .492, estadísticamente significativa-.

${ }^{21}$ Es pertinente recordar que el análisis factorial es un método estadístico inductivo diseñado para reducir un gran número de variables a componentes más pequeños mediante la búsqueda de intercorrelaciones entre grupos de esos juegos de variables. Véase Pallant (2007: 179) y de Vaus (2002: 134). Para el caso de las variables de identidad yucateca, el análisis factorial reveló, de las 12 preguntas o ítems originales, tres dimensiones que comprenden cinco preguntas, como se indica anteriormente. Por supuesto, el cuestionario completo está disponible a solicitud.

${ }^{22}$ El Tratado TLCAN fue signado por México, Estados Unidos y Canadá en 1992 y entró en vigor el primero de enero de 1994.

23 Hay limitaciones de espacio para describir ampliamente el desarrollo de las variables mencionadas así como su codificación. Cualquier información adicional relativa a las escalas factoriales y al cuestionario original de la Encuesta Yucatán está disponible a solicitud.

${ }^{24}$ La regresión es una técnica estadística usada para explorar y cuantificar la relación entre una variable dependiente y una o más variables independientes o predictoras (Landero y González, 2006: 343). El método usual de estimación de un modelo de regresión es el de mínimos cuadrados ordinarios, según el tipo de variable que se utilice.

${ }^{25}$ Traducción del autor.

26 Se agruparon los encuestados en tres niveles educativos: aquellos con educación primaria o menor nivel educativo - grados $1-6-, \mathrm{N}=128$; aquellos con educación secundaria y bachillerato $-7-12$, de aquí en adelante también denominados como medio-, $\mathrm{N}=164$; y aquellos con educación de nivel superior o alto nivel educativo - tecnológica, vocacional, licenciatura y posgrado-, $\mathrm{N}=101$.

${ }^{27}$ Quizá una muestra mayor hubiese convertido estas relaciones en estadísticamente significativas al nivel .05 y superior que al nivel actual -.157 y .165, respectivamente-

${ }^{28}$ Es necesario recalcar que para la muestra total las variables regionalismo y actitudes hacia Estados Unidos son predictores estadísticamente significativos para actitudes hacia el TLCAN al nivel 05 y superior; 
en el caso de actitudes hacia las privatizaciones, etnocentrismo e internacionalismo también son predictivas y estadísticamente significativas al nivel .05 y superior. Los resultados están disponibles a solicitud aunque por razones de espacio no se incluyen aquí.

${ }^{29}$ Entre aquellos de bajo nivel educativo, su identidad regional (beta =.322) y actitudes hacia Estados Unidos (beta $=.276$ ) están vinculadas con actitudes hacia el TLCAN al nivel de significancia de .05 o superior. Entre los de estrato medio, regionalismo (beta $=.206$, significante al nivel .05) y actitudes hacia Estados Unidos (beta $=.459$, significante al nivel .05 o superior) moldean las actitudes yucatecas hacia el TLCAN.

\section{Bibliografía}

Alberro, Solange (1994), "Yucatán: Una peculiaridad no desmentida”, Historia mexicana, vol. XLIII, núm. 3, enero-marzo, pp. 371-372.

Alisky, Marvin (1980), "The Relations of the State of Yucatan and the Federal Government of Mexico, 1823-1978", en Moseley, Edward H. y Edward D. Terry (eds.), Yucatán: A World Apart, Tuscaloosa: The University of Alabama Press, pp. 245-263.

Ard, Michael (2003), An Eternal Struggle: How the National Action Party Transformed Mexican Politics, Westport: Praeger Publishers

Baker, Andy (2006), "Why is Voting Behavior so Regionalized in Mexico? Political Discussion and Electoral Choice in 2006", artículo presentado en la 2006 Annual Meeting of the American Political Science Association, Philadelphia.

Baklanoff, Eric N. (2008), "Introduction: Yucatán since the 1982 Mexican Debt Crisis," en Baklanoffy, Eric N. y Edward H. Moseley (eds.), Yucatán in an Era of Globalization, Tuscaloosa: The University of Alabama Press, pp.1-19.

Barking, David y Timothy King (1970), Regional Economic Development: The River Basin Approach in México, Cambridge: Cambridge University Press.
Bassols Batalla, Ángel (1979), Formación de regiones económicas en México, México: Universidad Nacional Autónoma de México.

Beer, Caroline C. (2003), Electoral Competition and Institutional Change in Mexico, Notre Dame: University of Notre Dame Press.

Bruhn, Kathleen (1999), "The Resurrection of the Mexican Left in the 1997 Elections: Implications for the Party System", en Domínguez, Jorge I. y Alejandro Poiré (eds.), Towards Mexico's Democratization: Parties, Campaigns, Elections and Public Opinion, Nueva York: Routledge, pp. 88-113.

Bruhn, Kathleen (2004), "The Making of the Mexican President, 2000: Parties, Candidates, and Campaign Strategy", en Domínguez, Jorge I. y Chappell Lawson (eds.), Mexico's Pivotal Democratic Election. Candidates, Voters, and the Presidential Campaign of 2000, Stanford y La Jolla: Stanford University Press/Center for U.S.Mexican Studies, pp. 123-156.

Buendía, Jorge (1996), "Economic Reform, Public Opinion, and Presidential Approval in Mexico, 19881993", en Comparative Political Studies, vol. 29, núm.5, octubre, pp. 566-591.

Bunce, Valerie (2000), "Comparative Democratization. Big and Bounded Generalizations", en Comparative Political Studies, vol. 33, núm. 6/7, agosto-septiembre, pp. 703-734.

Cady, Frederic (2003), "Down but not Out: The PRI Survives Mexico's Democratization (How SocioDemographic Factors and Geography Relate to the PRI's Electoral Strength)", artículo presentado en la Annual Meeting for the Southwestern Political Science Association, San Antonio, TX (abril).

Camp, Roderic Ai (1999), Politics in Mexico: The Decline of Authoritarianism, Oxford: Oxford University Press.

Camp, Roderic Ai (2004), "Citizen Attitudes towards Democracy and Vicente Fox's Victory in 2000", en Domínguez, Jorge I. y Chappell Lawson (eds.), Mexico's Pivotal Democratic Election. Candidates, Voters, and the Presidential Campaign of 2000, Stanford y La Jolla: 
Stanford University Press/Center for U.S.-Mexican Studies, pp. 25-46.

Camp, Roderic Ai (2007), Politics in Mexico. The Democratic Consolidation, Oxford: Oxford University Press.

Cansino, César (2000), La transición mexicana 1977-2000, México: Centro de Estudios de Política Comparada.

Canto Sáenz, Rodolfo (2001), Del henequén a las maquiladoras. La política industrial en Yucatán 1984-2001, México: INAP/Universidad Autónoma de Yucatán.

Carrillo Aronte, Ricardo (1972), "La estrategia del desarrollo regional de México", en Soto Mora, Consuelo (ed.), Memoria del coloquio sobre planificación regional, México: Universidad Autónoma de México, pp.15-24.

Casar, María Amparo y Wilson Peres (1988), El Estado empresario en México: iagotamiento o renovación? México: Siglo XXI.

Cornelius, Wayne, Todd A. Eisenstadt y Jane Hindley (eds.) (1999), Subnational Politics and Democratization in Mexico, La Jolla: Center for U.S.- Mexican Studies/ University of California, San Diego.

De Vaus, David (2002), Analyzing Social Science Data. 50 Key Problems in Data Analysis, Londres: Sage Publications.

Díaz-Cayeros, Alberto (2004), "Decentralization, Democratization, and Federalism in Mexico", en Middlebrook, Kevin J. (ed.), Dilemmas of Political Change in Mexico. San Diego: Center for U.S.-Mexican Studies/University of California, pp. 198-236.

Domínguez, Jorge I. (2004). "Conclusion: Why and How Did Mexico's 2000 Presidential Election Matter?", en Domínguez, Jorge I. y Chappell Lawson (eds.), Mexico's Pivotal Democratic Election. Candidates, Voters, and the Presidential Campaign of 2000, Stanford y La Jolla: Stanford University Press/Center for U.S.-Mexican Studies, pp. 321-344.

Domínguez, Jorge I. y James A. McCann (1996), Democratizing Mexico: Public Opinion and Electoral Choices, Baltimore: John Hopkins University Press.

Dumond, Don E. (1997), The Machete and the Cross: Campesino Rebellion in Yucatan. Lincoln: University of Nebraska Press.
Figueroa Magaña, Jorge Enrique (2009), The Role of Subnational Identities in Shaping Mass Citizen Attitudes towards Economic Globalization and Economic Reforms: The Case of the State of Yucatan, (tesis de doctorado inédita), Universidad de Kentucky.

Figueroa Magaña, Jorge Enrique (2013), “El país como ningún otro. Un análisis empírico del regionalismo yucateco", manuscrito aceptado para su publicación en Estudios Sociológicos, vol. XXXI, núm. 91, eneroabril.

Hernández Valdez, Alfonso (2000), “Las causas estructurales de la democracia local en México, 19891998", Política y gobierno, vol. 7, núm. 1, pp. 101-144.

Hiskey, Jonathan T., y Shaun Bowler (2005), "Local Context and Democratization in Mexico", en American Journal of Political Science, vol. 49, núm.l, enero, pp. 57-71.

Huesca-Dorantes, Patricia (2003), The Emergence of Multiparty Competition in Mexican Politics, Burlington: Ashgate Publishing Company.

Instituto Federal Electoral (2006), Estadísticas 2006, México: IFE. 〈http://www.ife.org.mx/portal/site/ ifev2/Menu_Principal/?vgnextoid=bl4cf485le2ee010 VgnVCM1000002c01000aRCRD> [14 de septiembre de 2012].

Klesner, Joseph L. (1993), "Modernization, Economic Crisis, and Electoral Alignment in Mexico", en Mexican Studies/Estudios Mexicanos, vol. 9, núm. 2, verano, pp. 187-223.

Klesner, Joseph L. (1995), "The 1994 MexicanElection: Manifestation of a DividedSociety?", en Mexican Studies/Estudios Mexicanos, vol. 1l, núm. 1, primavera, pp. 137-149.

Klesner, Joseph L. (200la), "Economic Integration and Subregional Electoral Dynamics in Mexico", en Bailey, John (ed.), U.S.-Mexican Economic Integration: NAFTA at the Grassroots: Local Impact of Trade and Integration in Mexico and the United States, Austin: LBJ School of Public Affairs/University of Texas Press, pp. 167-197. 
Klesner, Joseph L. (200lb), “Realignment or Dealignment? Consequences of Economic Crisis and Restructuring for the Mexican Party System", en Cook, María Lorena, Kevin Middlebrook y Juan Molinar Horcasitas (eds.), The Politics of Economic Reestructuring: State-Society Change in Mexico, San Diego: Center for U.S. Mexican Studies/ University of California, San Diego, pp. 159-191.

Klesner, Joseph L. (2004), "The Structure of the Mexican Electorate: Social, Attitudinal, and Partisan Bases of Vicente Fox's Victory", en Domínguez, Jorge I. y Chappell Lawson (eds.), Mexico's Pivotal Democratic Election. Candidates, Voters, and the Presidential Campaign of 2000, Stanford y La Jolla: Stanford University Press/ Center for U.S.-Mexican Studies, pp. 91-122.

Klesner, Joseph L. (2007), “The 2006 Mexican Elections: Manifestation of a Divided Society?", en PS: Political Science and Politics, vol. 40, enero, pp. 27-32.

Klesner, Joseph L. (2012), "Regionalism in Mexican Electoral Politics", en Camp, Roderic Ai (ed), The Oxford Handbook of Mexican Politics, Oxford: Oxford University Press, pp. 622-648.

Landero Hernández, René y Mónica T. González Ramírez (2006), Estadística con SPSS y metodología de la investigación, México: Trillas.

Lawson, Chappell (2004a), “Mexico's Great Debates: The Televised Candidate Encounters of 2000 and Their Electoral Consequence", en Domínguez, Jorge I. y Chappell Lawson (eds.), Mexico's Pivotal Democratic Election. Candidates, Voters, and the Presidential Campaign of 2000, Stanford y La Jolla: Stanford University Press/ Center for U.S.-Mexican Studies, pp. 211-242.

Lawson, Chappell (2004b), "Television Coverage, Vote Choice, and the 2000 Campaign”, en Domínguez,Jorge I. y Chappell Lawson (eds.), Mexico's Pivotal Democratic Election. Candidates, Voters, and the Presidential Campaign of 2000, Stanford y La Jolla: Stanford University Press/ Center for U.S.-Mexican Studies, pp. 187-210.

Lawson, Chappell (2006), "Preliminary Findings from the Mexico 2006 Panel Study Memo \#1: Blue States and Yellow States", manuscrito inédito disponible en 〈http://web.mit.edu/clawson/www/polisci/research/ mexico06/Papers.html> [28 de mayo de 2012].

Lawson, Chappell (2007), "How Did We Get Here?, Mexican Democracy after the 2006 Elections", en PS: Political Science and Politics, vol. 40, enero, pp. 45-48.

Mabry, Donald J. (1973), Mexico's Acción Nacional: A Catholic Alternative to Revolution, Syracuse: Siracusa University Press.

Magaloni, Beatriz (1999), "Is the PRI Fading? Economic Performance, Electoral Accountability, and Voting Behavior in the 1994 and 1997 Elections", en Domínguez, Jorge I. y Alejandro Poiré (eds.), Towards Mexico's Democratization: Parties, Campaigns, Elections and Public Opinion, Nueva York: Routledge, pp. 203-236.

Markusen, Ann (1987), Regions: The Economics and Politics of Territory, Totowa: Rowman and Littlefield, Publishers.

McCann, James A. (1998), "The Changing Mexican Electorate: Political Interest, Expertise and Party Support in the 1980s and 1990s", en Serrano, Mónica (ed.), Governing Mexico: Political Parties and Elections, Londres: University of London, pp. 15-37.

Montalvo Ortega, Enrique e Iván Vallado Fajardo (1997), Yucatán: sociedad, economía, política y cultura, México: Universidad Nacional Autónoma de México.

Moreno, Alejandro (1999), "Campaign Awareness and Voting in the 1997 Mexican Congressional Elections", en Domínguez, Jorge I. y Alejandro Poiré (eds.), Towards Mexico's Democratization: Parties, Campaigns, Elections and Public Opinion, Nueva York: Routledge, pp. 114-146.

Moreno, Alejandro (2004), "The Effects of Negative Campaigns on Mexican Voters", en Domínguez, Jorge I. y Chappell Lawson (eds.), Mexico's Pivotal Democratic Election. Candidates, Voters, and the Presidential Campaign of 2000, Stanford y La Jolla: Stanford University Press/ Center for U.S.-Mexican Studies, pp. 243-268.

Moseley H., Edward y Edward D. Terry (eds.) (1980), Yucatán: A World Apart. Tuscaloosa: The University of Alabama Press. 
Needler, Martin C. (1995), Mexican Politics: The Containment of Conflict, Westport: Praeger Publishers.

Otero, Gerardo (2004), “Mexico's double movement: neoliberal globalism, the state and civil society" en Otero, Gerardo (ed), Mexico in transition: Neoliberal globalism, the state and civil society, Londres: Zed Books, pp. 1-17

Pallant, Julie (2007), SPSS Survival Manual. A Step by Step Guide to Data Analysis Using SPSS for Windows Third Edition, Berkshire: McGraw-Hill.

Poiré, Alejandro (1999), "Retrospective Voting, Partisanship, and Loyalty in Presidential Elections: 1994", en Domínguez, Jorge I. y Alejandro Poiré (eds.), Towards Mexico's Democratization: Parties, Campaigns, Elections and Public Opinion, Nueva York: Routledge, pp. 24-56.

Rodríguez, Victoria E. y Peter M. Ward (eds.) (1995), Opposition Government in Mexico, Albuquerque: The University of New Mexico Press.

Ross, Marc Howard (1997), "Culture and Identity in Comparative Political Analysis", en Lichbach, Mark I. y Alan S. Zuckerman (eds.), Comparative Politics. Rationality, Culture, and Structure. Cambridge: Cambridge University Press, pp. 42-80.

Rubio, Luis (1998), "Coping with Political Change", en Kaufman Purcell, Susan y Luis Rubio, (eds.) Mexicounder Zedillo, Boulder: Lynne Rienner Publishers, pp. 5-36.
Shirk, David A. (2005), Mexico's New Politics: The PAN and Democratic Change, Boulder: Lynne Rienner Publishers. Sierra Villarreal, José Luis (1995), Las concertacesiones en Yucatán. Conservadurismo y antidemocracia, Mérida: Centro de Estudios de la Democracia.

Sierra Villarreal, José Luis (2000), La plutocraciaen Yucatán, 2 vol., Mérida: Compañía Editorial de la Península/ Fundación Colosio-Yucatán, A.C.

Sierra Villarreal, José Luis, Antonio Paz Pineda y Guadalupe Huchim Koyoc (1986), Política y poder en Yucatán, Mérida: Academia Yucatanense de Ciencias y Artes/Instituto de Investigaciones SocialesUNAM.

Spalding, Rose (1999), "Party Politics in Yucatan: Regional Political Strategy in Mexico", artículo presentado para el $57^{\text {th }}$ Annual Meeting of the Midwest Political Association, Chicago, IL, abril 15-17).

Spalding, Rose (2000), "Opposition Politics, Party Pluralism, and Electoral Democratization in Yucatan", artículo presentado para el XXII International Congress of the Latin American Studies Association, Miami FL, marzo 16-18.

Spalding, Rose (2001), "Opposition, Social Policy and Sub-National Politics in Mexico: The 2000 Elections in Yucatan", artículo presentado para el 59 $9^{\text {th }}$ Annual Meeting of the Midwest Political Science Association, Chicago, IL, abril 19-22. 
Tabla 1. Modelo de atención cultural de lealtad panista —modelo de efectos directos-.

\begin{tabular}{|c|c|c|c|c|c|c|c|c|}
\hline & \multicolumn{2}{|c|}{ Muestra total } & \multicolumn{2}{|c|}{ Primaria } & \multicolumn{2}{|r|}{ Medio } & \multicolumn{2}{|c|}{ Superior } \\
\hline Variables predictivas: & & Correlaciones & & Correlaciones & & Correlaciones & & Correlaciones \\
\hline (Coeficientes Beta) & Beta & de orden cero & Beta & de orden cero & Beta & de orden cero & Beta & de orden cero \\
\hline \multicolumn{9}{|l|}{ Cultural } \\
\hline Regionalismo & -.008 & .034 & .093 & .157 & -.074 & -.046 & -.040 & .019 \\
\hline Etnocentrismo & .019 & .043 & .132 & .165 & -.023 & .012 & -.075 & -.051 \\
\hline $\begin{array}{l}\text { Actitudes hacia } \\
\text { Estados Unidos }\end{array}$ & .012 & .075 & .025 & .069 & .049 & .107 & -.050 & .142 \\
\hline $\mathrm{R}^{2}$ ajustadas & \multicolumn{2}{|r|}{.027} & \multicolumn{2}{|r|}{.016} & \multicolumn{2}{|r|}{.026} & \multicolumn{2}{|r|}{.066} \\
\hline Valor $\mathrm{F}$ & \multicolumn{2}{|r|}{2.689} & \multicolumn{2}{|r|}{1.292} & \multicolumn{2}{|r|}{1.647} & \multicolumn{2}{|r|}{2.149} \\
\hline Nivel de significancia & \multicolumn{2}{|r|}{.021} & \multicolumn{2}{|r|}{.275} & \multicolumn{2}{|r|}{.153} & \multicolumn{2}{|r|}{.069} \\
\hline
\end{tabular}

Nota: los coeficientes de regresión están ajustados para reflejar los efectos de las variables de tipo policy —actitudes hacia el TLCAN y privatizaciones-.

Tabla 2. Efectos de política pública — policy - de lealtad panista: actitudes como predictores de lealtad panista.

\begin{tabular}{|c|c|c|c|c|c|c|c|c|c|c|c|c|}
\hline & \multicolumn{3}{|c|}{ Muestra total } & \multicolumn{3}{|c|}{ Primaria } & \multicolumn{3}{|c|}{ Medio } & \multicolumn{3}{|c|}{ Superior } \\
\hline $\begin{array}{l}\text { Variables } \\
\text { predictivas: }\end{array}$ & & & Correlaciones & & & Correlaciones & & & Correlaciones & & & Correlaciones \\
\hline $\begin{array}{l}\text { (Coeficientes } \\
\text { Beta) }\end{array}$ & Beta & & de orden cero & Beta & & de orden cero & Beta & & de orden cero & Beta & & de orden cero \\
\hline \multicolumn{13}{|l|}{$\begin{array}{l}\text { Actitudes } \\
\text { sobre políticas } \\
\text { públicas } \\
\text { (policy) }\end{array}$} \\
\hline TLCAN & .185 & * & $.198 *$ & .215 & * & $.209 *$ & .178 & * & $.194 *$ & .255 & * & $.279 *$ \\
\hline Privatizaciones & .071 & & .095 & -.015 & & -.014 & .133 & & $.171^{*}$ & .208 & & $.237 *$ \\
\hline $\mathrm{R}^{2}$ ajustadas & \multicolumn{3}{|r|}{.040} & \multicolumn{3}{|c|}{.031} & \multicolumn{3}{|r|}{.037} & \multicolumn{3}{|r|}{.073} \\
\hline Valor F & \multicolumn{3}{|r|}{3.147} & \multicolumn{3}{|c|}{1.518} & \multicolumn{3}{|c|}{1.765} & \multicolumn{3}{|c|}{2.036} \\
\hline $\begin{array}{l}\text { Nivel de } \\
\text { significancia }\end{array}$ & \multicolumn{3}{|r|}{.005} & \multicolumn{3}{|c|}{.181} & \multicolumn{3}{|r|}{.113} & \multicolumn{3}{|c|}{.072} \\
\hline
\end{tabular}

Nota: los coeficientes de regresión están ajustados para reflejar los efectos de las variables económicas (ingreso y tipo de empleo). Los resultados de estas dos variables no se muestran. El asterisco denota significancia al nivel .05 y superior. 


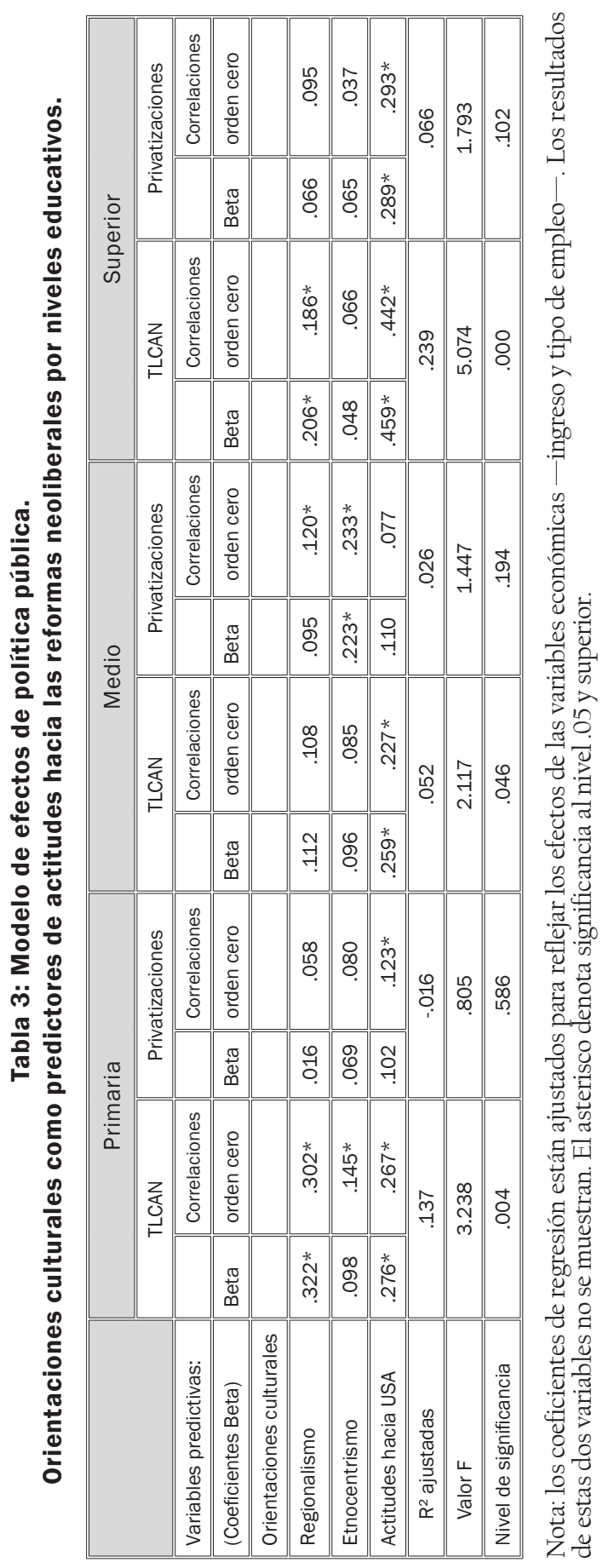

